$1 \ldots$

\title{
Possível utilização de canabinoides na Doença de Alzheimer: uma revisão
}

\section{Possible use of cannabinoides in Alzheimer's Disease: a review}

\author{
Vagner Fagnani Linartevichi ${ }^{1 *}$, Rafael de Morais Cury ${ }^{1}$, Francisney Pinto Nascimento ${ }^{2}$ \\ ${ }^{1}$ Programa de Pós-Graduação em Biociências da Universidade Federal da Integração Latino-Americana - UNILA \\ ${ }^{2}$ Docente do Programa de Pós-Graduação em Biociências Universidade Federal da Integração Latino-Americana - \\ UNILA
}

\section{ARTICLE INFO \\ Article history: \\ Received 18 November 2021 \\ Revised 09 February 2021 \\ Accepted 23 February 2021 \\ Available online 15 June 2021 \\ Blind reviews}

\section{Keywords: \\ Cannabinoids \\ Endocannabinoids \\ Neurodegeneration}

\begin{abstract}
The present study aimed to conduct an exploratory review of the literature on the use of cannabinoids for the treatment of $A D$ in order to discuss the medicinal use of these substances for possible future treatments. Dissertation review of the literature in which the articles were searched using two variables, namely disease (Alzheimer's Disease, Alzheimer Dementia) and intervention (Cannabi, Marihuana, Cannabis sativa, sativa, indicas, Cannabis). The results shown in the literature suggest that phytocannabinoids can be a useful tool in AD, mainly because isolated studies show the suppression of factors related to the neurobiology of the disease. Apparently, the association of CBD and THC appears to be more promising than their use alone. In this context, the clinical use of cannabinoids should be considered as a possible pharmacological tool in the treatment of several diseases, including AD. In fact, more clinical studies should be encouraged.
\end{abstract}

\section{Palavras-chave: \\ Canabinoides \\ Endocanabinoides \\ Neurodegeneração}

\footnotetext{
* Corresponding author at: linartevichi@gmail.com https://orcid.org/0000-0003-4893-7623
}

\section{RESUMO}

O presente estudo teve por objetivo realizar uma revisão exploratória da literatura sobre o uso de canabinoides para o tratamento da DA no intuito de discutir a utilização medicinal destas substâncias para possíveis tratamentos futuros. Revisão dissertativa da literatura no qual os artigos foram buscados por meio de duas variáveis sendo elas a doença (Alzheimer's Disease, Alzheimer Dementia) e a intervenção (Cannabi, Marihuana, Cannabis sativa, sativa, indicas, Cannabis). Os resultados mostrados na literatura sugerem que os fitocanabinoides podem ser uma ferramenta útil na DA, principalmente pelo fato de estudos isolados mostrarem a supressão dos fatores relacionados com a neurobiologia da doença. Aparentemente a associação de CBD e THC parece ser mais promissora do que seu uso isoladamente. Neste contexto, o uso clínico de canabinoides deve ser considerado como uma possível ferramenta farmacológica no tratamento de diversas doenças incluindo a DA. Para tanto, mais estudos clínicos devem ser estimulados.

https://doi.org/10.35984/fjh.v3i2.365

(C) 2021 The Authors. Published by FAG Journal of Health. This is na open accss article under the CC BY-NC-ND licence (http://creativecommons.org/licenses/by-nc-nd/4.0/) 


\section{Introdução}

O interesse em doenças senis tem crescido nos últimos anos devido ao fato do aumento da expectativa de vida. Várias são as demências que acometem esta faixa etária incluindo a Doença de Alzheimer (DA), demência vascular, demência paralítica, demência por corpos de Lewy, demência induzida por monóxido de carbono e demência traumática (PARK, KIM, 2014). Desde 1995 a incidência da DA vem aumentando em detrimento à demência vascular, assim a DA tem chamado mais a atenção da comunidade científica (HAN et al., 2017). A DA é causada por uma neurodegeneração de neurônios corticais, existe a presença de placa amiloigênicas, hiperfosforilação da proteína TAU e um processo neuroinflamatório. Os principais sintomas são perda de memória, declínio cognitivo e alterações comportamentais. Do ponto de vista histopatológico há uma atrofia cerebral, a presença de corpos senis e uma neurofibromatose (NFT) (OIDE et al., 2006). O déficit cognitivo induzido pela DA gera um importante impacto social na vida dos pacientes. Assim, tratamento que pudessem ativamente produzir uma melhora cognitiva afetariam positivamente o prognostico da DA. Neste sentido, muitos estudos tem sido focados em novos produtos farmacêuticos capazes de restabelecer a capacidade cognitiva destes pacientes, incluindo os derivados do gênero Cannabis (HAN et al., 2017).

A Cannabis contém pelo menos 113 tipos de canabinoides. O THC ( $\Delta$-tetrahidrocanabinol), o CBD (canabidiol), o CBG (canabigerol) e o CBN (canabinol) são os canabinoides mais conhecidos e diversos estudos tem sido conduzidos a partir deles (PATRICIA, JOSEPH, 2019). Diversos grupos de pesquisa tem encontrado efeitos clínicos importante com a utilização destes canabinoides tais como: alívio da dor, diminuição da inflamação, efeito neuroprotetor, vasoprotetor e tratamento da insônia (HA, 2018). Apesar deste achados, as pesquisas envolvendo canabinoides encontra diversas limitações, uma vez que, trata-se de um assunto delicado envolvendo diversas esferas da sociedade incluindo a criminal.

Dentro dos fitocanabinoides presentes na Cannabis o $\triangle 9$-THC e o CBD são os mais estudados. O CBD não apresenta os mesmos efeitos psicoativos do $\triangle 9$-THC (FELLERMEIER et al., 2001). Estes efeitos produzidos pelo $\triangle 9$-THC derivam em parte da ativação dos receptores CB1 (receptores canabinoides tipo 1), os quais são encontrados abundantemente em todo o SNC (sistema nervoso central). Altas densidades dos receptores CB1 já foram descritas nos núcleos dos gânglios basais, substância negra parte reticulada, segmentos internos e externos do globo pálido. No hipocampo, particularmente no giro denteado e no cerebelo altas densidades dos receptores CB1 já foram descritas (PERTWEE, 2008). Esta localização dos receptores no SNC sugere que há um envolvimento deste sistema na modulação da memória, emoção, dor e movimento (MAROON, BOST, 2018). Os receptores do tipo CB2 também já foram localizados no SNC, sendo descrita como uma alta densidade em várias regiões do córtex e do hipocampo. As principais funções descritas para este receptor até o momento estão relacionadas ao processo inflamatório, modulação da liberação de citocinas e sobrevivência e diferenciação de células neuronais e não neuronais (SVÍŽENSKÁ et al., 2009).

Neste contexto, o presente estudo teve por objetivo realizar uma revisão exploratória da literatura sobre o uso de canabinoides para o tratamento da DA no intuito de discutir a utilização medicinal destas substâncias para possíveis tratamentos futuros.

\section{Metodologia}

O presente estudo buscou artigos publicados até dezembro de 2020 em duas bases de dados EMBASE e PUBMED. Foram aceitos apenas os trabalhos que apresentaram o texto completo em idioma inglês. Os artigos foram buscados por meio de duas variáveis sendo elas a doença (Alzheimer's Disease, Alzheimer Dementia) e a intervenção (Cannabi, Marihuana, Cannabis sativa, sativa, indicas, Cannabis). Apenas foram selecionados artigos que tratavam da eficácia do uso de Cannabis sativa e seus componentes em pacientes com DA ou testes pré-clínicos preditivos da DA.

\section{Resultados e discussão}

Um total de 1283 artigos foram encontrados sendo que destes 1145 foram excluídos por não trazerem os termos de busca no título ou no resumo, não estarem relacionados com DA ou seu tratamento por meio de canabinoides. Outros 65 artigos foram excluídos por focarem em estudos in vitro. $\mathrm{Na}$ sequência 42 artigos foram excluídos por duplicidade do grupo de pesquisa. Outros 14 não foram possíveis acessar o texto completo. Restando então 17 trabalhos que foram utilizados para a presente discussão.

Dois estudos realizados em camundongos testaram o efeito da aplicação do CBD em animais injetados com o peptídeo $\beta$-amiloide $(\mathrm{A} \beta$ ) humano no hipocampo dorsal. Os resultados mostraram que o CBD diminuiu significativamente a expressão do GFAP, RNAm e proteína relacionados à iNOS e IL-1 $\beta$ (indicando a diminuição de padrões moleculares relacionados à neuroinflamação) (ESPOSITO et al., 2007). Além disso, estes efeitos foram confirmados com ou sem a administração de antagonistas PPAR $\gamma$. Neste sentido, o estudo sugere que o CBD diminuiu o dano inflamatório neural e facilitou a neurogênese hipocampal (GIUSEPPE et al., 2011).

A administração de canabinoides em modelo animal da DA demonstrou que o agonista do receptor CB1 foi capaz de prevenir a hiperfosforilação da proteína TAU, além de diminuir as mudanças celulares e comportamentais induzidas pela administração da proteína A $\beta$ (IUVONE et al., 2009). Além disso, a ativação microglial induzida pela $\mathrm{AD}$ foi inibida por agonistas CB1. Também foi notada uma menor alteração na morfologia celular no SNC, diminuição da liberação do fator de necrose tumoral e menor taxa de lesão mitocondrial nestes animais (RAMIREZ et al., 2005). Adicionalmente, $\Delta 9$ THC diminuiu a agitação motora dos ratos, evento comum em pacientes com DA (SALAZAR et al., 2009).

$\mathrm{O}$ tratamento baseado em fitocanabinoides em uma combinação 1:1 de CBD e $\Delta 9$-THC produziu em humanos um efeito neuroprotetor, estes efeitos mediados pela ativação dos receptores CB1 e CB2 (VALDEOLIVAS et al., 2012).

Além disso, o CBD tem sido correlacionado com a modulação de diferentes receptores fora do SNC. Os receptores da serotonina já foram correlacionados aos efeitos terapêuticos do CBD. Em um modelo animal já foi sugerida a capacidade do CBD estimular a neurogênese. A ação neuroprotetora do CBD durante um dano cerebral induzido por hipóxia já foi correlacionado com a participação dos receptores A2 da adenosina. A ativação deste tipo de receptor induzida pelo CBD resultou em efeitos anti-inflamatórios e imunossupressor. Adicionalmente, em um modelo animal de DA, a administração de CBD inibiu o efeito da gliose reativa

FAG Journal of Health - ISSN 2674-550X, 2021, v.3, n.2, p.196 
e subsequente neurotoxicidade induzida pela $\beta$ A (SAGREDO et al., 2007).

Em um modelo animal de DA, o tratamento com $\Delta 9$ THC (3 $\mathrm{mg} / \mathrm{kg}$ ) uma vez ao dia por quatro semanas adicionados com um inibidor da COX2 reduziu o número de placas de $\beta$ A e degeneração neural. Já o tratamento com 2,5 $\mathrm{mg}$ de dronabinol (um análogo sintético do $\Delta 9$-THC) diariamente por duas semanas aumentos o escore neuropsiquiátrico de pacientes com DA, diminuindo os sintomas de agitação motora e noturna (WALTHER et al., 2006).

Ainda neste contexto, estudos de caso que compararam a evolução de lesões traumáticas no SNC relataram que, pacientes que afirmaram terem feito uso prévio de THC tiveram uma melhor evolução do processo inflamatório comparado com pacientes que não utilizaram a substância (SCHURMAN, LICHTMAN, 2017). A figura 1 faz um apanhado sobre os efeitos encontrados para os canabinoides em algumas células do sistema nervoso central (SNC).

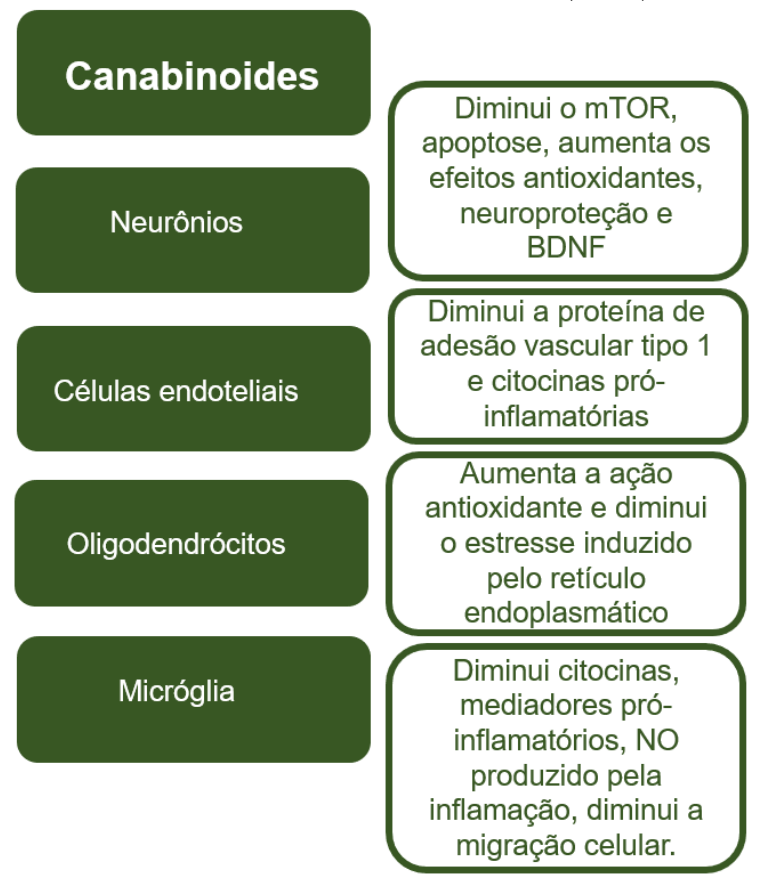

Figura 1 - Vários alvos celulares de ação dos canabinoides no sistema nervoso central (Adaptado de MAROON e BOST, 2018).

Do ponto de vista clínico, embora nenhum estudo relate que a Cannabis possa modificar, retardar ou curar a DA, várias publicações relatam que os canabinoides podem melhorar os sintomas neuropsiquiátricos relacionados a DA (tais como agitação, perda de peso e alterações do sono - além dos efeitos bioquímicos já relatados anteriormente) (INGLET et al., 2020)

Um estudo conduzido por Shelef e colaboradores (2016) observou a eficácia da administração de um óleo de Cannabis $(1,65 \%$ de $\Delta 9$-THC) no controle dos sintomas psicológicos e comportamentais da demência em 11 pacientes hospitalizados portadores da DA. Foi encontrada significativa melhora nos parâmetros neuropsiquiátricos na quarta semana de tratamento, não foi observada mudança no escore do mini mental.

Ruthirakuhan e colaboradores (2019) analisaram seis ensaios clínicos duplo-cegos randomizados de portadores da DA que receberam oralmente $\triangle 9$-THC, dronabinol ou nabilona para o controle da agressividade e agitação relacionadas à DA. Neste estudo, os autores não encontraram diferença significativa entre os grupos que receberam as drogas canabinoides, THC ou placebo. Entretanto, este e outros estudos encontraram que a administração desses compostos se mostraram seguros e bem tolerados em pacientes com DA (AHMED et al., 2015). Deste modo, os dados relacionados aos efeitos dos canabinoides no controle dos sintomas relacionados a demência ainda são escassos e merecem mais atenção.

De maneira indireta, diversos trabalhos já demonstraram os efeitos neuroprotetores do $\triangle 9$-THC, incluindo em modelos pré-clínicos da DA. Esse efeito parece ser diretamente relacionado à uma modulação de células neurais e não neurais. Os mecanismos desta modulação incluem a regulação da transmissão glutamatérgica e da plasticidade sináptica, regulação da resposta imune, liberação de mediadores inflamatórios, alteração do funcionamento do receptor NMDA do glutamato, canais de cálcio e sistemas antioxidantes (LIGRESTI et al., 2016).

Adicionalmente, sabe-se que doses moderadas de $\Delta 9$ THC afeta o sistema serotoninérgico, diminuindo a atividade do receptor 5HT3, mecanismo embasa um efeito antiemético deste canabinóide. Este achado pode ser útil no sentido de diminuir a náusea, quando comparado com tratamentos convencionais (VELENOVSKÁ, FISAR, 2007).

Sabemos ainda que pacientes com DA apresentam inúmeras alterações neuropsiquiátricas. A avaliação direta dessas condições em pacientes com demência e o efeito dos canabinoides ainda não foi realizada. No entanto de maneira indireta vários trabalhos já foram conduzidos. Sabemos que no SNC os antidepressivos aumentam as concentrações do BDNF (fator neurotrófico derivado do cérebro) e diminuem o processo inflamatório (MARESE et al., 2019, LINARTEVICHI et al., 2021, NESTLER et al., 2002). Neste sentido, Mecha e colaboradores (2012) observaram resultados semelhantes com o uso de canabinoides. Adicionalmente, a administração de CBD atenuou as respostas autonômicas evocadas pelo estresse, mostrando em modelos animais um efeito tipo ansiolítico e antidepressivo, ambos por meio da ativação dos receptores 5HT1a (URIBE-MARIÑO et al., 2012). Neste sentido, adicionalmente, o uso de canabinoides poderia atenuar os sintomas adjuntos à DA.

Com relação aos principais problemas relacionados ao uso de canabinoides foram relatados psicose, disforia e ansiedade apenas com o uso de altas doses de $\Delta 9-T H C$, de modo que, existem discussões para que o uso de CBD não seja mais considerado dopping em competições esportivas. Cabe ressaltar que nenhuma morte direta ou indireta foi atribuída pelo uso recreativo de canabinoides (KOPPEL et al., 2014; WADA, 2018).

Não existe consenso sobre a dose de canabinoides a ser utilizadas, sabe-se que a combinação de concentrações de CBD e $\triangle 9$-THC tem sido útil em alguns tratamentos, recomenda-se sempre a utilização das menores concentrações de 49 -THC possíveis (LIGRESTI et al., 2016; MAROON, BOST, 2018).

\section{Considerações finais}

Os canabinoides agem em diversos alvos tais como o processamento da $\beta \mathrm{A}$ e TAU, neuroinflamação, excitotoxicidade, estresse oxidativo e disfunção mitocondrial, processos íntimos ao mal prognóstico da DA. Sendo que, estudos in vitro $\mathrm{e}$ in vivo sugerem que os canabinoides atenuam esses eventos nocivos. 
O presente estudo sumarizou algumas evidências da utilização de canabinoides por pacientes portadores de DA. Fica claro que os estudos disponíveis são escassos. No entanto, há indícios sugestivos dos benefícios moleculares e comportamentais da administração de derivados da Cannabis em pacientes com DA. Não menos importante, acreditamos que mais estudos devem ser realizados com um número maior de pacientes para que dados mais robustos sejam produzidos afim de garantir a efetividade e segurança destes derivados assim como, dosagem, tempo de utilização. Testes clínicos com Cannabinoides, ou outros ativos, devem ser estimulados pelos governantes considerando que a DA trata-se de uma doença crônica e até o momento tratada de modo limitado pelos medicamentos disponíveis.

\section{Conflito de interesse}

Os autores declaram não haver conflito de interesse.

\section{Agradecimento}

Os autores agradecem o apoio da Universidade Federal da Integração Latino Americana, em especial ao Programa de Pós Graduação em Biociências pela colaboração com o presente projeto de Pós-Doutorado.

\section{Referências}

AHMED A, ELSEN G, COLBERS A, et al. Safety, pharmacodynamics, and pharmacokinetics of multiple oral doses of delta-9-tetrahydrocannabinol in older persons with dementia. Psychopharmacology, v. 232, p. $2587-$ 2595, 2015. http://doi.org/10.1007/ s00213-015-3889-y

ESPOSITO G, SCUDERI C, SAVANI C, STEARDO L, DE FILIPPIS D, COTTONE P, et al. Cannabidiol in vivo blunts $\beta$-amyloid induced neuroinflammation by suppressing IL-1 $\beta$ and iNOS expression. British Journal of Pharmacology, v. 151, n. 8, p. 1272-1279, 2007, http://doi.org/10.1038/sj.bjp.0707337

FELLERMEIER M, EISENREICH W, BACHER A, ZENK MH, Biosynthesis of cannabinoids. Eur J Biochem, v. 268, p. 1596-1604, 2001, http://doi.org/10.1046/j.14321033.2001.02030.x

GIUSEPPE E, CATERINA S, MARTA V, GIUSEPPINA IT, VALENTINA L, DANIELE DF, et al. Cannabidiol reduces $\mathrm{A} \beta$-induced neuroinflammation and promotes hippocampal neurogenesis through PPAR $\gamma$ involvement. PLOS ONE, v.6, n. 12, p. 28668, 2011, http://doi.org/10.1371/journal.pone.0028668

HA SY. Perception Survey of the Use of Medical Marihuana Targeting Cancer-Patients and each of their Protectors. 2018, Korea:Hansung Univ. [Dissertation]

HAN DY, PARK NE, KIM SH, JEONG DG. The Effect of Oral Administration of Herbal Medicines on Memory in Alzheimer's Disease Animal Models: A Review of Animal Study Reports Published in Korea. JPPKM, v. 28, n. 4, p. 359-371, 2017, http://doi.org/10.3831/KPI.2019.22.030

INGLET S, WINTER B, YOST SE, ENTRINGER S, LIAN A, BIKSACKY M, PITT RD, MORTENSEN W. Clinical Data for the Use of Cannabis-Based Treatments: A Comprehensive Review of the Literature. Annals of Pharmacotherapy, v. 54, n. 11, p. 1109-1143, 2020. http://doi.org/10.1177/1060028020930189.

IUVONE T, ESPOSITO G, DE FILIPPIS D, SCUDERI C, STEARDO L. Cannabidiol: A promising drug for neurodegenerative disorders? CNS Neuroscience \&
Therapeutics, v.15, p. 65-75, 2009, http://doi.org/10.1111/j.1755-5949.2008.00065.x

KOPPEL BS, BRUST JCM, FIFE T, BRONSTEIN J, YOUSSOF S, GRONSETH G, et al. Systematic review: Efficacy and safety of medical marijuana in selected neurologic disorders, Report of the Guideline Development Subcommittee of the American Academy of Neurology. Neurology, v. 82, p. 1556-1563, 2014, http://doi.org/10.1212/WNL.0000000000000363

LIGRESTI A, DE PETROCELLIS L, DI MARZO V. From phytocannabinoids to cannabinoid receptors and endocannabinoids: Pleiotropic physiological and pathological roles through complex pharmacology. Physiological Reviews, v. 96, p. 1593-1659, 2016, http://doi.org/10.1152/physrev.00002.2016

LINARTEVICHI, VF.; FROZA, MG.; CURY, RM.; NASCIMENTO, FP. Potencial uso da psilocibina no tratamento da depressão: uma revisão. Brazilian Journal of Development, v. 7, n. 3, p. 32270-32288, 2021, https://doi.org/10.34117/bjdv7n3-783

MARESE, A.; FICAGNA, E.; PARIZOTTO, R.; LINARTEVICHI, V. Principais mecanismos que correlacionam a microbiota intestinal com a patogênese da depressão. Fag Journal of Health, v. 1, n. 3, p. 232-239, 20 out. 2019. https://doi.org/10.35984/fjh.v1i2.40

MAROON J., BOST J. Review of the neurological benefits of phytocannabinoids. Surgical Neurology International, v. 9, p. 91-104, 2018. https://doi.org/10.4103/sni_45_18

MECHA M, TORRAO AS, MESTRE L, CARRILLO-SALINAS FJ, MECHOULAM R, GUAZA C. Cannabidiol protects oligodendrocyte progenitor cells from inflammation-induced apoptosis by attenuating endoplasmic reticulum stress. Cell Death Disease, v. 3, p. 331, 2012, http://doi.org/10.1038/cddis.2012.71

NESTLER EJ, BARROT M, DILEONE RJ, EISCH AJ, GOLD SJ, MONTEGGIA LM. Neurobiology of depression. Neuron, v. 34, p. 13-25, 2002, http://doi.org/10.1016/s0896-6273(02)00653-0

OIDE T, KINOSHITA T, ARIMA K. Regression stage senile plaques in the natural course of alzheimer's disease. Neuropathology and Applied Neurobiology, v. 32, n. 5, p. 539-556, 2006, http://doi.org/10.1111/j.13652990.2006.00767.x

PARK MS, KIM YM. Study on Syndrome Differentiation of Dementia. JPPKM, v. 28, n. 3, p. 251-262, 2014, http://doi.org/10.15188/kjopp.2014.06.28.3.251

PATRICIA R, JOSEPH S. Medical Marijuana and pain management. Peer-reviewed excellence in life care planning, v. $19, \quad$ n. $3, \quad$ p. 20-25, 2019, http://doi.org/10.1016/j.disamonth.2016.05.014

PERTWEE RG. The diverse CB1 and CB2 receptor pharmacology of three plant cannabinoids: $\Delta$ 9-tetrahydrocannabinol, cannabidiol and $\Delta$ 9-tetrahydrocannabivarin. British Journal of Pharmacology, v. 153, p. 199-215, 2008. http://doi.org/10.1038/sj.bjp.0707442

RAMIREZ BG, BLAZQUEZ C, GOMEZ DEL PULGAR T, GUZMAN M, DE CEBALLOS ML. Prevention of Alzheimer's disease pathology by cannabinoids: Neuroprotection mediated by blockade of microglial activation. The Journal of Neuroscience, v. 25, p. 19041913, 2005. https://doi.org/10.1523/jneurosci.454004.2005 
RUTHIRAKUHAN M, LANCTÔT KL, VIEIRA D, HERRMANN N. Natural and synthetic cannabinoids for agitation and aggression in Alzheimer's disease: a metaanalysis. The Journal of Clinical Psychiatry, v. 80, n. 18, p. 12617, 2019. http://doi.org/10.4088/JCP.18r12617

SAGREDO O, RAMOS JA, DECIO A, MECHOULAM R, FERNANDEZ-RUIZ J. Cannabidiol reduced the striatal atrophy caused 3-nitropropionic acid in vivo by mechanisms independent of the activation of cannabinoid, vanilloid TRPV1 and adenosine A2A receptors. European Journal of Neuroscience, v.26, p. 843-851, 2007. http://doi.org/10.1111/j.1460-9568.2007.05717.x.

SALAZAR M, CARRACEDO A, SALANUEVA IJ, HERNANDEZ-TIEDRA S, EGIA A, LORENTE M, et al. TRB3 links ER stress to autophagy in cannabinoid anti-tumoral action. Autophagy, v. 5, p. 1048-1049, 2009, http://doi.org/10.4161/auto.5.7.9508

SCHURMAN LD, LICHTMAN AH. Endocannabinoids: A promising impact for traumatic brain injury. Frontiers in $\begin{array}{llllll}\text { Pharmacology, } & \text { v. } & 8, & \text { p. } & 69, & 2017 \text {, }\end{array}$ http://doi.org/doi.org/10.3389/fphar.2017.00069

SHELEF A, BARAK Y, BERGER U, et al. Safety and efficacy of medical cannabis oil for behavioral and psychological symptoms of dementia: an-open label, addon, pilot study. Journal of Alzheimer's Disease, v. 51, p. 15-19, 2016. http://doi.org/10.3233/JAD-150915

SVÍŽENSKÁ, I., DUBOVÝ, P., \& ŠULCOVÁ, A. Cannabinoid receptors 1 and 2 (CB1 and $\mathrm{CB} 2)$, their distribution, ligands and functional involvement in nervous system structures: a short review. Pharmacology Biochemistry, and Behaviour, v. 90, p. 501-511, 2009. http://doi.org/10.1016/j.pbb.2008.05.010

URIBE-MARIÑO A, FRANCISCO A, CASTIBLANCO-URBINA MA, TWARDOWSCHY A, SALGADO-ROHNER CJ, CRIPPA, J, et al. Anti-Aversive Effects of Cannabidiol on Innate Fear-Induced Behaviors Evoked by an Ethological Model of Panic Attacks Based on a Prey vs the Wild Snake Epicrates cenchria crassus Confrontation Paradigm. Neuropsychopharmacology, v. 37, p. 412-421, 2012, http://doi.org/10.1038/npp.2011.188

VALDEOLIVAS S, SATTA $\mathrm{V}$, PERTWEE RG, FERNANDEZ-RUIZ J, SAGREDO O. Sativex-like combination of phytocannabinoids is neuroprotective in malonate-lesioned rats, an inflammatory model of Huntington's disease: Role of $\mathrm{CB} 1$ and $\mathrm{CB} 2$ receptors. ACS Chem Neurosci, v. 3, p. 400-406, 2012, http://doi.org/10.1021/cn200114w

VELENOVSKÁ M, FISAR Z. Effect of cannabinoids on platelet serotonin uptake. Addiction Biology, v.12, p. 158166, 2007, http://doi.org/10.1111/j.13691600.2007.00065.x

WADA.-AMA.org. The world anti-doping code international standard prohibited list, January 2018. Available from: https://www.wada-ama.org/sites/default/ files/prohibited_list_2018_en.pdf. [Last accessed on 2021 Jan 12].

WALTHER S, MAHLBERG R, EICHMANN U, KUNZ D. Delta-9-tetrahydrocannabinol for nighttime agitation in severe dementia. Psychopharmacology, v. 185, p. 524528, 2006, http://doi.org/10.1007/s00213-006-0343-1 